\title{
Coordination characteristics of three N,N'-di(azol-1-yl) methanes; substituent effects on infrared absorption and structure of the Group VI metal carbonyl derivatives *
}

\author{
Kom-Bei Shiu and Kuen-Song Liou \\ Department of Chemistry, National Cheng Kung University, Tainan 70101 (Taiwan)
}

Yu Wang, Ming-Chu Cheng and Gene-Hsiang Lee

Department of Chemistry, National Taiwan University, Taipei 10764 (Taiwan)

(Received October 17, 1992; in revised form December 11, 1992)

\begin{abstract}
Compounds $\left[\mathrm{M}\left(\mathrm{H}_{2} \mathrm{CAz}_{2}\right)(\mathrm{CO})_{4}\right](\mathrm{M}=\mathrm{Cr}, \mathrm{Mo}$, or $\mathrm{W})$, were obtained readily and similarly by refluxing the equivalent amounts of $\mathrm{N}, \mathrm{N}^{\prime}$-di(azol-1-yl)methanes, $\mathrm{H}_{2} \mathrm{CAz}_{2}\left(\mathrm{Az}=\right.$ pyrazol-1-yl $(\mathrm{Pz})$, 3,5-dimethylpyrazol-1-yl $\left(\mathrm{Pz}^{\prime}\right)$ or 3,4,5-trimethylpyrazol-1-yl $\left(\mathrm{Pz}^{\prime \prime}\right)$ with $\left[\mathrm{M}(\mathrm{CO})_{6}\right](\mathrm{M}=\mathrm{Cr}, \mathrm{Mo}$, or $\mathrm{W})$ in a mixed solvent system of 1,2-dimethoxyethane and tetrahydrofuran. Under phase transfer catalysis conditions this gives rise to the new nitrogen-bidentate ligand, $\mathrm{H}_{2} \mathrm{CPz}_{2}^{\prime \prime}$, by reaction of $\mathrm{HPz}^{\prime \prime}$ with methylene chloride. IR absorption bands in the carbonyl region indicate that no regular trends can be found for different substituents on the pyrazolyl ring. This irregularity is attributed to flexibility of the boat conformation formed from the metal centre and the ligand, which is supported by comparative structure details of $\left[\mathrm{Mo}\left(\mathrm{H}_{2} \mathrm{CPz}_{2}^{\prime}\right)(\mathrm{CO})_{4}\right]$, reported previously, $\left[\mathrm{W}\left(\mathrm{H}_{2} \mathrm{CPz}_{2}^{\prime \prime}\right)(\mathrm{CO})_{4}\right](6)$ and $\left[\mathrm{W}\left(\mathrm{H}_{2} \mathrm{CPz}_{2}^{\prime}\right)(\mathrm{CO})_{4}\right](7): 6$, triclinic, $P \overline{1}, a=8.403(3), b=9.589(3), c=13.310(3) \AA$, $\alpha=94.476(22), \beta=102.837(21), \gamma=110.355$ $(25)^{\circ}, Z=2, R=0.024, R_{\mathrm{w}}=0.023$, based on 3070 with $I>2 \sigma(I) .7$, monoclinic, $P 21 / c, a=9.178(3), b=15.885(9), c=12.394(10)$ $\AA, \beta=108.64(9)^{\circ}, Z=4, R=0.030, R_{\mathrm{w}}=0.030$, based on 2625 reflections with $I>2.5 \sigma(I)$.
\end{abstract}

\section{Introduction}

The syntheses and applications of neutral poly(azol1-yl)alkanes, $\mathrm{R}_{n} \mathrm{C}(\mathrm{Az})_{4-n}(\mathrm{R}=\mathrm{H}$, alkyl, or aryl; $\mathrm{HAz}=$ di-, tri- or tetra-azole; $n=2,3$, or 4$)$, as multidentate ligands with either main-group or transition-metal atoms have been extensively studied [1-4]. Especially recently, ligands with $\mathrm{Az}=$ pyrazol-1-yl $(\mathrm{Pz})$ or 3,5-dimethylpyrazol-1-yl ( $\left.\mathrm{Pz}^{\prime}\right)$ have been the most vigorously developed. From the literature, it appears obvious, as one would expect, that ligands with more methyl-group substituents should have greater electron-donating ability, a feature previnusly reported for 1,10phenanthroline (phen) (or 2,2'-bipyridine (bpy)) and the relevant substituted derivatives [5]. However, we

\footnotetext{
Correspondence to: Dr. K.-B. Shiu.

* Part of synthetic result is taken from K.-S. Liou, M. Sc. Thesis, National Cheng Kung University, Tainan, 1988.
}

wish to present IR evidence that no regular trends are in fact observable for $\left[\mathrm{Cr}\left(\mathrm{H}_{2} \mathrm{CAz}_{2}\right)(\mathrm{CO})_{4}\right],\left[\mathrm{Mo}\left(\mathrm{H}_{2}\right.\right.$ $\left.\left.\mathrm{CAz}_{2}\right) \mathrm{i}(\mathrm{CO})_{4}\right]$, or $\left[\mathrm{W}\left(\mathrm{H}_{2} \mathrm{CAz}_{2}\right)(\mathrm{CO})_{4}\right]$. This unexpected irregularity can be rationalized by comparing the structural details of $\left[\mathrm{Mo}\left(\mathrm{H}_{2} \mathrm{CPz}_{2}^{\prime}\right)(\mathrm{CO})_{4}\right]$, reported in 1989 [6], $\left.\mathrm{W}\left(\mathrm{H}_{2} \mathrm{CPz}_{2}^{\prime}\right)(\mathrm{CO})_{4}\right]$ and $\left[\mathrm{W}\left(\mathrm{H}_{2} \mathrm{CPz}_{2}^{\prime \prime}\right)\right.$ $(\mathrm{CO})_{4}$ ], where $\mathrm{Pz}^{\prime \prime}$ is 3,4,5-trimethylpyrazol-1-yl (Fig. 1).

\section{Experimental section}

All operations were performed by the usual Schlenk techniques [7], using deoxygenated, dry solvents and gases. IR spectra, calibrated with polystyrene, were recorded on a Hitachi Model 260-30 instrument. Abbreviations are as follows: vs, very strong; s, strong; m, medium and sh, shoulder. NMR spectra were obtained on a Bruker WP-100 $\left({ }^{1} \mathrm{H}, 100 \mathrm{MHz}\right)$ FT-NMR spectrometer. Chemical shifts ( $\delta$ in ppm, $J$ in herz) are 


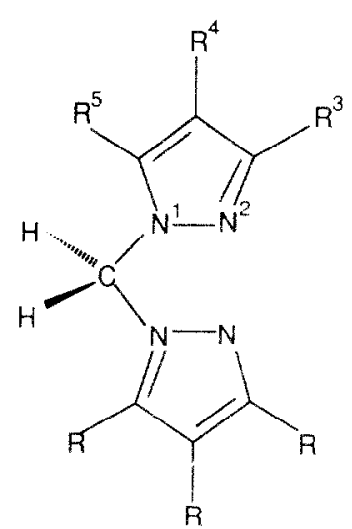

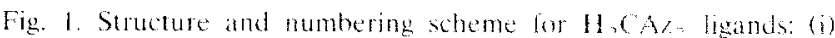
$3 \mathrm{C}-\mathrm{R}=4 \mathrm{C}-\mathrm{R}=5 \mathrm{C} \cdot \mathrm{R}=\mathrm{H} ; \mathrm{H}, \mathrm{CA}=\mathrm{H}(\mathrm{P} 2$, (i) $3 \mathrm{C}-\mathrm{R}=5 \mathrm{C}$

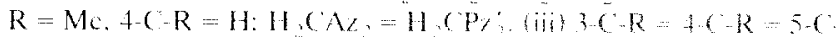
$\mathrm{R}=\mathrm{Me}: \mathrm{H}, \mathrm{CA} /=\mathrm{H}(\mathrm{P} \%$

defined as positive downfield or negative upfield relative to internal SiMe, (TMS) standard; abbreviations are s. singlet; d. doublet; br, unresolved multiplet or two overlapped singlets. Elemental analysis was performed by the Microanalytical Service of the Department of Chemistry, National Cheng Kung University. Melting points were determined with a Mel-Temp apparatus (Laboratory Devices) and are not corrected.

3,4,5-Trimethylpyrazole ( $\mathrm{HPz}$ ") was prepared from 3-methylpentane-2,4-dione [8] by following Vogel's procedure [9]. HPz", a white solid with a typical vield of $78 \%$, mp. $139-140{ }^{\circ} \mathrm{C}$. Anal. Found: $\mathrm{C} \quad 65.53 ; \mathrm{H}$. 9.18: N. 25.18. $\mathrm{C}_{6} \mathrm{H}_{10} \mathrm{~N}$ calc: $\mathrm{C} .65 .43 ; \mathrm{H} .9 .15 ; \mathrm{N}$, $25.42 \%$ 'H NMR $\left(23^{\circ} \mathrm{C}, \mathrm{CDCl}\right.$. $\left.100 \mathrm{MHz}\right), 3$ and 5-methyl groups, $02.18(6 \mathrm{H}, \mathrm{s})$; 4-methyl group, 1.89 $(3 \mathrm{H}, \mathrm{s}) ; \mathrm{N}-\mathrm{H} .11 .11(1 \mathrm{H}, \mathrm{s}))$. The ligand $\mathrm{H}, \mathrm{CP}_{2}$," was then prepared with the same procedure as for $\mathrm{H}, \mathrm{CPZ}_{2}$ ? [3]: H.CP2", a white floppy solid, yicld $85 \%$ mp. 146-147 C. Anal. Found: C. 66.98; H, 8.60: N. 24.13. $\mathrm{C}_{13} \mathrm{H}_{2,1} \mathrm{~N}_{4}$ calc: $\mathrm{C}, 67.20 ;$ H. 8.68; N. 24.12\%, 'H NMR $\left.\left(23^{\circ} \mathrm{C}, \mathrm{CDCl}_{3}, 100\right) \mathrm{MHz}\right): 3-$ and 5 -melhy groups, 8 $2.12(6 \mathrm{H}, \mathrm{s}) .2 .32(6 \mathrm{H}, \mathrm{s}): 4$-methyl group $1.64(6 \mathrm{H}, \mathrm{s})$ $\mathrm{CH}, 6.04(2 \mathrm{H}, \mathrm{br})$

\subsection{Preparation of $/ \mathrm{Cr}\left(\mathrm{H}_{2} \mathrm{CP}_{2}\right) /(\mathrm{CO}) /(1)$}

A mixture of $\mathrm{H}_{2} \mathrm{CPZ}_{2}(0.74 \mathrm{~g}, 5.00 \mathrm{mmol})$, prepared from HPZ (Aldrich) by the literature procedure [3], and $\left[\mathrm{Cr}(\mathrm{CO})_{6}\right](1.10 \mathrm{~g}, 5.00 \mathrm{mmol})$ in $40 \mathrm{ml}$ of 1,2 -dimethoxyethane (DME) and $10 \mathrm{ml}$ of tetrahydrofuran (THF) was refluxed for $24 \mathrm{~h}$. The solvents were then removed under vacuum. Recrystallization from acetone $/ \mathrm{MeOH}$ gave the vellow product $(1.30 \mathrm{~g} .87 \%)$. Anal. Found: $\mathrm{C}, 42.30 ; \mathrm{H}, 2.65 ; \mathrm{N}, 17.95, \mathrm{C}_{11} \mathrm{H}_{3} \mathrm{CrN}_{4} \mathrm{O}_{4}$ calc: C. 42.32; H, 2.58; N. $17.95 \%$ ' H NMR $123^{\circ} \mathrm{C}$. acetone- $d_{6}, 100 \mathrm{MH}$ ): hydrogen atoms on the ring-3 and -5 positions. $\delta 8.14(2 \mathrm{H}, \mathrm{d} .3(\mathrm{H} . \mathrm{H})=2.4) .8(0)$
(2H, d. $9(\mathrm{HH})=19)$, hydrogen atoms on the ring-4 position. 6.48 (2H. 1): $\mathrm{CH}, 6.64(2 \mathrm{H}, 6 \mathrm{r})$. IR (CH, ( ) $)$ "(CO). $2000 \mathrm{~m}$. $180 \mathrm{ks}, 1 \times 72 \mathrm{sh}$. 1832 and IR (KBr):

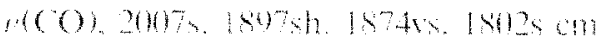

\section{2. Preparation of / Worth, (P) , $16(0), /(2)$}

This milky yellow compound was obtained in $85 \%$ yield by an analogous procedure (ca. 3 h). Anal. Found:

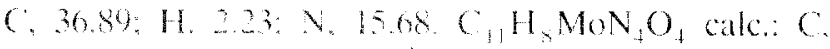
$37.16 \%$ H. $2.26 \%, 15.74$ "II NMR $23 \mathrm{C} . \mathrm{CDCl}_{3}, 100$ $\mathrm{MH}$ ): hydrogen atoms on the ring-3 and -5 positions.

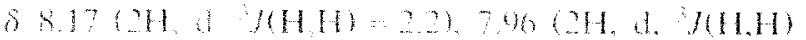
1.95 hydrogen atoms on the ring-t powition, $0.480 \mathrm{H}$. 1): $\mathrm{CH}, 6.74(2 \mathrm{H}, \mathrm{hr})$ IR $(\mathrm{MCON}) \mathrm{aCO}, 2014 \mathrm{~m}$,

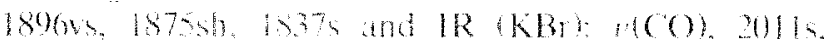
$1915 \mathrm{sh} .1872 \mathrm{w} .1865 \mathrm{~cm}$

\section{Preparation of $/ \mathrm{H} / \mathrm{H}, \mathrm{CP}=2)(\mathrm{CO} / \mathrm{O} /(3)$}

This yellow compound was obtancd in $78 \%$ yield by an analogrous procedure (ad $2+$ in) Anal Found: $C$.

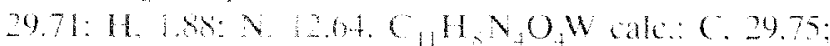
H. 1.82; N. 12.62\% H NMR $23 \mathrm{C} .(\mathrm{CDCl}, 100 \mathrm{MHz})$ : hydrogen atoms on the ring-3 and 5 positions, is 8.22 $(2 \mathrm{H}, \mathrm{d}, \quad 3(\mathrm{H}, \mathrm{H})=2.3), 8.09(2 \mathrm{H}, \mathrm{d}, \quad y(\mathrm{H}, \mathrm{H})=2 \mathrm{H})$ hydrogen atoms on the ring-4 position. $0.53(2 \mathrm{H}, \mathrm{t})$ $(\mathrm{H}, 6.80(2 \mathrm{H} . \mathrm{hr})$. IR (MerN): $(\mathrm{CO}) 2004 \mathrm{~m} .1879 \mathrm{~m}$ $1827 \mathrm{~s}$ and IR (KBr): $/(\mathrm{CO})$. 2004s 1897sh, 1802vs. $1702 \mathrm{~cm}$ !

\section{Preparation of / $\mathrm{CrH}, \mathrm{CPz} / \mathrm{lCO} / \mathrm{C} / \mathrm{A})$}

This compound was obtained in $78 \%$ yield by an analogous prosedure (at. 24 h) from a mixture of $(0.27$

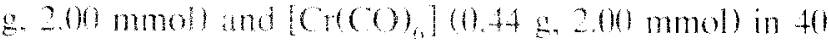
$\mathrm{ml}$ of DME and $10 \mathrm{ml}$ of THF. Recrytallization from $\mathrm{CH}, \mathrm{Cl}, \mathrm{MeOH}$ gate the pale orange-yellow product $(0.64 \mathrm{~g} .81 \%)$. Anal Found $(51.36 ; 14.5 .12 ; \mathrm{N}, 14.23$. $\mathrm{C}_{17} \mathrm{H}_{21} \mathrm{CNN}_{4} \mathrm{O}$ cake: C. $31.51 \% \mathrm{H}, 5.0 \% ; \mathrm{N}, 14.14 \%$ 'H NMR $23 \mathrm{C}, \mathrm{CDCl}, 100 \mathrm{MHz})$ : 3 and 5 -methyl groups. $82.276 \mathrm{H}$ \& $2.456 \mathrm{H}$ s $)$; tmethy group, $1.886 \mathrm{H}$,

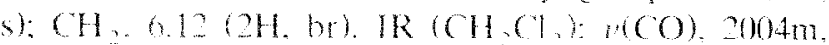
$1884 \mathrm{vs}, 1869 \mathrm{sh}$. 1827s and IR $(\mathrm{KBr})$ : $10 \mathrm{CO}$. 200ss. $188451.19678 \mathrm{~s} .1817 \mathrm{sm}$

\subsection{Preparation of $/ \mathrm{Mol} \mathrm{H}_{2}\left(\mathrm{P}=3 / \mathrm{CO} \mathrm{C}_{4} /(5)\right.$}

This pak yollow compound was obtained in $85 \%$ yield by an analogous procedure $1, a .25 \mathrm{~h}$ ). Anal. Found: $\mathrm{C}$, to.21: H. 455 N. 12.70. $\mathrm{C}_{1} \mathrm{H}_{20} \mathrm{MON}_{4} \mathrm{O}_{4}$ calc: $\mathrm{C}, 46.37 \% 14,4.58: \mathrm{N} .12 .73 \%, \mathrm{H}$ NMR $23^{\circ} \mathrm{C}$. $\left.\mathrm{CDCl}_{3}, 160 \mathrm{MH}^{2}\right)$ : 3 and 5 -methyl groups $82.3066 \mathrm{H}$. s). $2.3960 \mathrm{H}$, s): tmethy group. $1.89(6 \mathrm{H}, \mathrm{s}), \mathrm{CH}, 0.32$

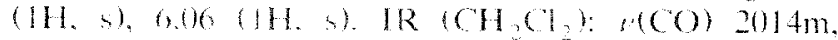
$1895 \mathrm{~s}, 1874 \mathrm{sh}$. 1832s and $\mathrm{IR}(\mathrm{KBH}),(\mathrm{CO}), 201 \mathrm{~s}$.

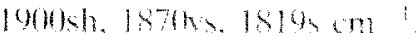




\subsection{Preparation of $\left[\mathrm{W}\left(\mathrm{H}_{2} \mathrm{CPz}_{2}^{\prime \prime}\right)(\mathrm{CO})_{4}\right]$ (6)}

This yellow compound was obtained in $83 \%$ yield by an analogous procedure ( $c a .24 \mathrm{~h}$ ). Anal. Found: C, $38.44 ; \mathrm{H}, 3.81 ; \mathrm{N}, 10.48 . \mathrm{C}_{17} \mathrm{H}_{20} \mathrm{~N}_{4} \mathrm{O}_{4} \mathrm{~W}$ calc.: $\mathrm{C}, 38.65$; $\mathrm{H}, 3.82 ; \mathrm{N}, 10.61 \% .{ }^{1} \mathrm{H}$ NMR $\left(23^{\circ} \mathrm{C}, \mathrm{CDCl}_{3}, 100 \mathrm{MHz}\right)$ : 3 - and 5-methyl groups, $\delta 2.31(6 \mathrm{H}, \mathrm{s}), 2.40(6 \mathrm{H}, \mathrm{s})$; 4-methyl group, $1.90(6 \mathrm{H}, \mathrm{s}) ; \mathrm{CH}_{2}, 6.31(1 \mathrm{H}, \mathrm{s}), 6.06$ $(1 \mathrm{H}, \mathrm{s})$. IR $\left(\mathrm{CH}_{2} \mathrm{Cl}_{2}\right): \nu(\mathrm{CO}), 2003 \mathrm{~m}, 1877 \mathrm{vs}, 1867 \mathrm{sh}$, 1822s and IR (KBr): $\nu(\mathrm{CO}), 1995 \mathrm{~s}, 1874 \mathrm{sh}, 1857 \mathrm{vs,}$ $1810 \mathrm{~s} \mathrm{~cm}^{-1}$.

2.7. X-Ray diffraction study of $\left[\mathrm{W}\left(\mathrm{H}_{2} \mathrm{CPz}_{2}^{\prime \prime}\right)(\mathrm{CO})_{4}\right]$ (6) and $\left.\left[\mathrm{W}^{\left(\mathrm{H}_{2} \mathrm{CPz}\right.} \mathrm{C}_{2}^{\prime}\right)(\mathrm{CO})_{4}\right](7)$

Crystals of 6 and 7 were grown from $\mathrm{CH}_{2} \mathrm{Cl}_{2} /$ hexane at room temperature. Gencral procedures and listings of programs were given previously [6]. Absorption correction was performed on both structures using $\psi$ scans. Related crystal data (Table 1), final coordinates of the non-hydrogen atoms (Table 2), and selected bond lengths and bond angles (Table 3) are reported. The anisotropic displacement coefficients of the atoms, the $\mathrm{H}$-atom coordinates and structural factors are available from the authors. The ORTEF plots for 6 and 7 with the relevant numbering scheme are drawn in Figs. 2 and 3, respectively.

\section{Results and discussion}

We have prepared $\left[\mathrm{M}\left(\mathrm{H}_{2} \mathrm{CPz}_{2}\right)(\mathrm{CO})_{4}\right]$ and $\left[\mathrm{M}\left(\mathrm{H}_{2}-\right.\right.$ $\left.\left.\mathrm{CPz}_{2}^{\prime \prime}\right)(\mathrm{CO})_{4}\right](\mathrm{M}=\mathrm{Cr}, \mathrm{Mo}$, or $\mathrm{W})$ under similar reaction conditions to those used for $\left[\mathrm{M}\left(\mathrm{H}_{2} \mathrm{CPz}_{2}^{\prime}\right)(\mathrm{CO})_{4}\right]$ [10]. These compounds are well characterized as cis$\left[\mathrm{M}\left(\eta^{2}-\left(\mathrm{H}_{2} \mathrm{CAz}_{2}\right)\right)(\mathrm{CO})_{4}\right]$ by elemental analysis, IR and NMR spectral results. Since the IR data of [M$\left.\left(\mathrm{H}_{2} \mathrm{CPz}_{2}\right)(\mathrm{CO})_{4}\right]$ are quite similar to those reported previously by Lobia and Bonati [4], it is thus clear that our reaction condition can apply in the synthesis of the metal carbonyl derivatives of all three types of $\mathrm{H}_{2} \mathrm{Az}_{2}$, whether the substituents on the pyrazolyl ring are hydrogen atoms or methyl groups (the condition Lobia and Bonati used [4] applied only for $\mathrm{H}_{2} \mathrm{CAz}_{2}=$ $\mathrm{H}_{2} \mathrm{CPz}_{2}$.)

TABLE 1. Crystal data for 6 and 7

\begin{tabular}{|c|c|c|}
\hline Compound & 6 & 7 \\
\hline Empirical formula & \multirow{2}{*}{$\begin{array}{l}\mathrm{C}_{17} \mathrm{H}_{20} \mathrm{~N}_{4} \mathrm{O}_{4} \mathrm{~W} \\
\text { yellow }\end{array}$} & $\mathrm{C}_{15} \mathrm{H}_{16} \mathrm{~N}_{4} \mathrm{O}_{4} \mathrm{~W}$ \\
\hline Colour & & yellow \\
\hline Crystal size (mm) & $0.30 \times 0.36 \times 0.42$ & $0.40 \times 0.30 \times 0.30$ \\
\hline Space group & \multirow[t]{2}{*}{ triclinic, $P \overline{1}$} & monoclinic, $P 2_{1} / c$ \\
\hline Unit cell dimensions & & \\
\hline$a, b, c, \AA$ & $8.403(3), 9.589(3), 13.310(3)$ & $9.178(3), 15.885(9), 12.394(10)$ \\
\hline$\alpha, \beta, \gamma, \operatorname{deg}$ & $94.476(22), 102.837(21), 110.355(25)$ & $90,108.68(4), 90$ \\
\hline Volume, $\AA^{3}$ & $966.0(5)$ & $1711.8(17)$ \\
\hline$Z$ & 2 & 4 \\
\hline Formula weight & 528.21 & 500.16 \\
\hline$D_{\text {calc }}, \mathrm{g} / \mathrm{cm}^{3}$ & 1.816 & 1.941 \\
\hline$h, k, l$ ranges & 9 to 9,0 to $11,-15$ to 15 & -10 to 10,0 to 18,0 to 14 \\
\hline Abs cor & \multicolumn{2}{|l|}{$\psi$ scan } \\
\hline Abs coeff, $\mathrm{mm}^{-1}$ & 6.13 & 1.94 \\
\hline Transm range & $0.4799-0.9995$ & $0.5436-0.9984$ \\
\hline$F(000)$ & 511.83 & 951.66 \\
\hline Diffractometer used & \multicolumn{2}{|c|}{ Nonius CAD4 } \\
\hline Radiation; $\lambda, \AA$ & \multicolumn{2}{|c|}{ Mo $\mathrm{K} \alpha ; 0.70930$} \\
\hline Temperature (K) & \multicolumn{2}{|c|}{297} \\
\hline Scan type & \multicolumn{2}{|l|}{$\theta / 2 \theta$} \\
\hline $2 \theta$ range, deg & $2-50$ & \\
\hline Scan speed, deg/min & \multicolumn{2}{|l|}{$1.43-10$} \\
\hline Std rflns & \multicolumn{2}{|c|}{$3 \mathrm{std} / 7200 \mathrm{sec}$} \\
\hline Decay; $\%$ & $\leq 1$ & $\leq 1$ \\
\hline No. of unique rflns & 3403 & 2992 \\
\hline No. of rflns $\left(N_{0}\right)$ used & 3070 with $l>2.0 \sigma(l)$ & 2625 with $I>2.5 \sigma(l)$ \\
\hline No. of atoms refined & 46 & 40 \\
\hline No. of params $\left(\mathrm{N}_{\mathrm{v}}\right)$ refined & 236 & 218 \\
\hline $\operatorname{Max} \Delta / \sigma$ ratio & 0.347 & 0.012 \\
\hline$R ; R_{\mathrm{w}} \mathrm{S}^{\mathrm{a}}$ & $0.024,0.023,2.77$ & $0.030,0.030,4.54$ \\
\hline Resid peak; hole e $/ \AA^{3}$ & $0.96,-0.96$ & $0.94,-1.60^{b}$ \\
\hline
\end{tabular}

${ }^{\mathrm{a}} \mathrm{S}=\left[\sum_{w}\left|F_{\mathrm{o}}-F_{\mathrm{c}}\right|^{2} /\left(\mathrm{N}_{\mathrm{o}}-\mathrm{N}_{\mathrm{v}}\right)\right]^{1 / 2}, w^{-1}=\sigma^{2}\left(F_{\mathrm{o}}\right),{ }^{\mathrm{b}}$ ghost peaks. 
TABLE 2. Fractional atomic coordinates and $B_{i s e}$ " for 6 and 7

\begin{tabular}{|c|c|c|c|c|}
\hline & $x$ & $y$ & $z$ & $B_{i\langle i\}}$ \\
\hline \multicolumn{5}{|c|}{ (a) Compound 6} \\
\hline$W$ & $0.99397(3)$ & $0.712160(24)$ & $0.271358(16)$ & $2.922(11)$ \\
\hline $\mathrm{Cl}$ & $0.8986(7)$ & $0.7500(6)$ & $0.3913(4)$ & $3.5(3)$ \\
\hline $\mathrm{C} 2$ & $1.0809(8)$ & $0.5843(6)$ & $0.3547(4)$ & $4.2(3)$ \\
\hline $\mathrm{C} 3$ & $0.7774(8)$ & $0.53860(7)$ & $0.2179(4)$ & $4.5(3)$ \\
\hline C4 & $1.0909(9)$ & $0.6439(6)$ & $0.1596(4)$ & $4.4(4)$ \\
\hline$C 5$ & $1.4110(7)$ & $0.9530(7)$ & $0.3420(4)$ & $3.8(3)$ \\
\hline C6 & $1.5108(7)$ & $1.1072(7)$ & $0.3612(4)$ & $3.9(3)$ \\
\hline$C 7$ & $1.3932(7)$ & $1.1775(6)$ & $0.3533(4)$ & $3(x)$ \\
\hline $\mathrm{CB}$ & $1.0613(7)$ & $1.0788(5)$ & $0.3119(4)$ & $3.0(3)$ \\
\hline C9 & $0.8000(7)$ & $0.8408(6)$ & $0.0747(4)$ & $3.4(3)$ \\
\hline $\mathrm{C} 10$ & $0.7939(7)$ & $0.9763(6)$ & $0.0463(4)$ & $3 . .3(3)$ \\
\hline C11 & $0.8932(7)$ & $1.0848(6)$ & $0.1307(4)$ & $3.4(3)$ \\
\hline C 12 & $1.4788(8)$ & $0.8243(8)$ & $0.3427(5)$ & $5.4(4)$ \\
\hline C13 & $1.7067(8)$ & $1.1797(8)$ & $0.3882(5)$ & $6.2(4)$ \\
\hline C 14 & $1.4211(8)$ & $1.3411(7)$ & $0.3664(5)$ & $5.3(4)$ \\
\hline C 15 & $0.7122(9)$ & $0.6866(7)$ & $0.0104(4)$ & $5.8(4)$ \\
\hline $\mathrm{C} 16$ & $0.6984(9)$ & $0.9947(7)$ & $\cdots 0.0575(4)$ & $5 .(x)$ \\
\hline $\mathrm{C} 17$ & $0.9330(10)$ & $1.2490(7)$ & $0.1450(15)$ & $5.9(5)$ \\
\hline NI & $1.2399(6)$ & $0.4256(5)$ & $0.3225(3)$ & $3.22(23)$ \\
\hline $\mathrm{N} 2$ & $1.2309(6)$ & $1.0679(5)$ & $0.3299(3)$ & $3.144(21)$ \\
\hline N3 & $0.9557(5)$ & $1.0172(4)$ & $0.2058(3)$ & $2.88(21)$ \\
\hline N4 & $0.8985(6)$ & $0.8647(5)$ & $0.1728(3)$ & $3.10(21)$ \\
\hline $\mathrm{Ol}$ & $0.8406(6)$ & $0.7585(5)$ & $0.460903)$ & $5.7(3)$ \\
\hline $\mathrm{O} 2$ & $1.1288(6)$ & $0.5016(5)$ & $0.4017(3)$ & $0.3(3)$ \\
\hline 03 & $0.6501(6)$ & $0.427+(5)$ & $0.1892(4)$ & $6.5(3)$ \\
\hline $\mathrm{O} 4$ & $1.1494(7)$ & $0.5957(5)$ & $0.1010(3)$ & $6,6(3)$ \\
\hline \multicolumn{5}{|c|}{ (b) Compound 7} \\
\hline W & $0.96959(4)$ & $0.098930(17)$ & $0.284295(24)$ & $2.452(11)$ \\
\hline $\mathrm{Nl}$ & $0.7589(7)$ & $0.0450(3)$ & $0.3142(4)$ & $2.5(3)$ \\
\hline$N_{2}$ & $0.8590(7)$ & $0.0277(3)$ & $0.1196(4)$ & $2.7(3)$ \\
\hline N.3 & $0.7139(7)$ & $-0.0369(3)$ & $0.2865(5)$ & $2.8(3)$ \\
\hline $\mathrm{N} 4$ & $0.7822(7)$ & $-0.0462(3)$ & $0.1186(4)$ & $2.8(3)$ \\
\hline $\mathrm{C}$ & $0.7915(9)$ & $-0.0863(4)$ & $0.224 !(6)$ & $3.1(4)$ \\
\hline C.1 & $1.1461(10)$ & $0.1482(4)$ & $0.2547(6)$ & $3.9(4)$ \\
\hline 01 & $1.2548(7)$ & $0.1830(4)$ & $0.2455(5)$ & $55(3)$ \\
\hline $\mathrm{C} 2$ & $1.1148(9)$ & $0.0027(4)$ & $0.3495(6)$ & $3.0(3)$ \\
\hline $\mathrm{O} 2$ & $1.2110(7)$ & $-0.0451(3)$ & $0.3865(5)$ & $4.9(3)$ \\
\hline $\mathrm{C} 3$ & $0.8656(10)$ & $0.2103(4)$ & $0.2238(6)$ & $3.7(4)$ \\
\hline 03 & $0.8279(8)$ & $0.2762(3)$ & $0.1930(5)$ & 6. $6(4)$ \\
\hline $\mathrm{C} 4$ & $1.0469(10)$ & $0.1505(4)$ & $0.4363(6)$ & $4 .(1) 5)$ \\
\hline $\mathrm{O} 4$ & $1.0937(7)$ & $0.1792(3)$ & $0.5245(4)$ & $5.8(4)$ \\
\hline $\mathrm{C} 11$ & $0.6017(9)$ & $0.1628(4)$ & $0.3988(6)$ & S. $(x)$ \\
\hline C12 & $0.6561(9)$ & $0.0773(4)$ & $0.3587(5)$ & $2.7(3)$ \\
\hline C13 & $0.5471(9)$ & $0.0154(4)$ & $0.3601(6)$ & $3.3(4)$ \\
\hline C.14 & $0.5874(9)$ & $-0.0563(4)$ & $0.3142(6)$ & $3.0(3)$ \\
\hline C 15 & $0.5155(10)$ & $-0.1422(5)$ & $0.2955(7)$ & $4.8(5)$ \\
\hline$C 21$ & $0.8939(11)$ & $0.1254(5)$ & $-0.0264(6)$ & $4.6(5)$ \\
\hline $\mathrm{C} 22$ & $0.8279(9)$ & $0.0489(5)$ & $0.0093(6)$ & $3,3(4)$ \\
\hline $\mathrm{C} 23$ & $0.7321(10)$ & $-0.011(5)$ & $-0.0605(6)$ & $4.1(4)$ \\
\hline $\mathrm{C} 24$ & $0.7027(10)$ & $-0.0694(5)$ & $0.0100(7)$ & $4.2(4)$ \\
\hline $\mathrm{C} 25$ & $0.6045(13)$ & $-0.1463(6)$ & $-0.01185(7)$ & $6.3(6)$ \\
\hline
\end{tabular}

${ }^{a} B_{\text {iso }}$ is the mean of the principal axes of the themal ellipsoid.

In the literature, numerous cis-disubstituted tetracarbonyl complexes of the group VI mctal atoms, $\left[\mathrm{M}(\mathrm{L}-\mathrm{L})(\mathrm{CO})_{4}\right]$, are known [11]. The four carbonyl stretching bands (i.e., four IR-active vibrations), which are usually displayed in these compounds have been assigned by Cotton and Kraihanzel on the basis of a simple group theory analysis [12]; two vibrations associated with the trans-carbonyl groups (assigned as $\mathrm{A}_{\text {, }}$ $\mathrm{CO}^{(1)}$ and $\mathrm{B}_{2} \mathrm{CO}^{(1)}$ ) occur at $186^{\circ}$ and $1825 \mathrm{~cm}^{\prime}$ and two other vibrations associated with the cis-carbonyl ligands (assigned as $\mathrm{A}_{1} \mathrm{CO}^{(2)}$ and $\mathrm{B}_{1} \mathrm{CO}^{(2)}$ ) occur at the higher wavenumbers of 2009 and $1897 \mathrm{~cm}^{-1}$ in $\mathrm{CH}_{2} \mathrm{Cl}_{2}$ for $\mathrm{L}-\mathrm{L}=$ bpy. By comparing either only one or all v(CO) values of $\left[\mathrm{M}\left(\mathrm{L}-\mathrm{L}_{-}\right)(\mathrm{CO})_{4}\right][11]$ or $[$ cis- $\mathrm{M}(\mathrm{L}$ $\left.\mathrm{L}_{2}(\mathrm{CO})_{2}\right][13]$, one can deduce the relative $\pi$-ateceptor ability of chelating ligands. L-L, giving an order such as bpy $<$ phen $<\mathrm{dmpe}<\mathrm{dppm}<\mathrm{dppe}<\mathrm{PF}_{3}$ (two $\mathrm{PF}_{\text {; }}$ ligands take the place of one chelate), where dmpe is 1,2-bis(dimethylphosphinolethane: dppm is his(diphenylphosphino)-methane and dppe is 1.2-bis(diphenylphosphinolethane. Since the ligands we used are also symmetrical like dppm or bpy, we first list the u(CO) values for $\left[\mathrm{M}\left(\mathrm{H}_{2} \mathrm{CAz}_{2}\right)(\mathrm{CO})_{4}\right](\mathrm{M}=\mathrm{Cr}$, Mo, or $\mathrm{W}$ : $\mathrm{A} z=\mathrm{P} z, \mathrm{Pz}^{\prime}$, or $\mathrm{P}_{z}{ }^{\prime \prime}$ (Table 4 ) and then compare one or all $\nu(\mathrm{CO})$ bands in the hope that effects of different substituents would be reflected in the values, a feature previously reported for phen (or bpy) and the relevant substituted derivatives [5]. In fact. as observed from these values, no regular trend for $\left[\mathrm{Cr}\left(\mathrm{H}_{2} \mathrm{CAz}_{2}\right)(\mathrm{CO})_{4}\right]$. $\left[\mathrm{Mo}\left(\mathrm{H}_{2} \mathrm{CAz}_{2}\right)(\mathrm{CO})_{4}\right]$, or $\left[\mathrm{W}\left(\mathrm{H}_{2} \mathrm{CAz}_{2}\right)(\mathrm{CO})_{4}\right]$, can he inferred taking account of experimental error, although one can conclude that the,$(\mathrm{CO})$ values of $\left[\mathrm{Mo}\left(\mathrm{H}_{2}-\right.\right.$ $\left.\left.\mathrm{CAz}_{2}\right)(\mathrm{CO})_{4}\right]$ are similar to those of $\left[\mathrm{Mo}(\mathrm{bpy})(\mathrm{CO})_{4}\right]$ and the $\pi$-aceptor ability of $\mathrm{I}_{2} \mathrm{CA}_{2}$ is similar to that of bpy (previously, Oro at al. suggested the similarity in $\pi$-acid ahility between $\mathrm{H}_{2} \mathrm{CP}_{2}$, and bpy on the basis of comparing the $v(\mathrm{CO})$ values of $\left[\mathrm{Rh}\left(\mathrm{L}_{-} \mathrm{L}\right)\left(\mathrm{CO}_{2}\right] \mathrm{ClO}_{4}\right.$ [14]). Quite obviously, when the substituents on the pyrazolyl rings are changed. the donor ability of the ligand is different from what would be expected. Since donor ability can be inferred from related structures, we decided to characterize at least two compounds with different substituents by X-ray crystallography and compare related structural features.

From Figs. 2 and 3 and Table 2. one can observe clearly that structures of both $\left.\left[\mathrm{W}_{\left(\mathrm{H}_{2}\right.} \mathrm{CPz}_{2}^{\prime \prime}\right)(\mathrm{CO})_{4}\right](6)$ and $\left[\mathrm{W}\left(\mathrm{H}_{2} \mathrm{CPZ}_{2}^{\prime}\right)\left(\mathrm{CO}_{4}\right](7)\right.$ are very similar to that of $\left[\mathrm{Mo}\left(\mathrm{H}_{2} \mathrm{CPZ}_{2}^{\prime}\right)(\mathrm{CO})_{4}\right](8)[6]$. in having a six-membered boat metallacycle, formed from $\mathrm{H}_{2} \mathrm{CA}_{2}$ and the metal atom, and two distorted cis-carbonyls. However. if four different planes are defined and calculated for 6-8, including two pyrazolyl-ring planes and two other oncs (for example. $W, N(1), N(4)$ in one plane and $C(8)$. $\mathrm{N}(2), N(3)$ in the other in 6 (1 ig. 2)), different structural features resulting from the methyl-group substitution can be recognized evidently. These two planes form an angle of $82.9^{\circ}$ in $6.85 .1^{\circ}$ in 7 , and $86.1^{\circ}$ in 8 while the two pyrazolyl-ring planes form an angle of 
TABLE 3. Selected bond lengths $(\AA)$ and angles $\left({ }^{\circ}\right)$ for 6 and 7

\begin{tabular}{lr}
\hline (a) Compound 6 & \\
(i) Bond lengths & \\
W-C(1) & $2.000(6)$ \\
W-C(2) & $1.935(5)$ \\
W-C(3) & $1.929(7)$ \\
W-C(4) & $2.015(6)$ \\
W-N(1) & $2.267(4)$ \\
W-N(4) & $2.270(4)$ \\
$\mathrm{C}(1)-\mathrm{O}(1)$ & $1.149(7)$ \\
$\mathrm{C}(2)-\mathrm{O}(2)$ & $1.170(7)$ \\
$\mathrm{C}(3)-\mathrm{O}(3)$ & $1.178(8)$ \\
$\mathrm{C}(4)-\mathrm{O}(4)$ & $1.157(7)$ \\
$\mathrm{C}(5)-\mathrm{C}(6)$ & $1.393(9)$ \\
$\mathrm{C}(5)-\mathrm{C}(12)$ & $1.484(8)$ \\
$\mathrm{C}(5)-\mathrm{N}(1)$ & $1.328(7)$ \\
$\mathrm{C}(6)-\mathrm{C}(7)$ & $1.368(8)$ \\
(ii) Bond angles & \\
$\mathrm{C}(1)-\mathrm{W}-\mathrm{C}(2)$ & $84.57(22)$ \\
$\mathrm{C}(1)-\mathrm{W}-\mathrm{C}(3)$ & $86.57(24)$ \\
$\mathrm{C}(1)-\mathrm{W}-\mathrm{C}(4)$ & $170.80(21)$ \\
$\mathrm{C}(1)-\mathrm{W}-\mathrm{N}(1)$ & $95.11(19)$ \\
$\mathrm{C}(1)-\mathrm{W}-\mathrm{N}(4)$ & $96.38(18)$ \\
$\mathrm{C}(2)-\mathrm{W}-\mathrm{C}(3)$ & $86.78(25)$ \\
$\mathrm{C}(2)-\mathrm{W}-\mathrm{C}(4)$ & $86.83(22)$ \\
$\mathrm{C}(2)-\mathrm{W}-\mathrm{N}(1)$ & $98.08(21)$ \\
$\mathrm{C}(2)-\mathrm{W}-\mathrm{N}(4)$ & $178.61(21)$ \\
$\mathrm{C}(3)-\mathrm{W}-\mathrm{C}(4)$ & $89.71(25)$ \\
$\mathrm{C}(3)-\mathrm{W}-\mathrm{N}(1)$ & $174.99(19)$ \\
$\mathrm{C}(3)-\mathrm{W}-\mathrm{N}(4)$ & $94.28(20)$ \\
$\mathrm{C}(4)-\mathrm{W}-\mathrm{N}(1)$ & $89.31(20)$ \\
$\mathrm{C}(4)-\mathrm{W}-\mathrm{N}(4)$ & $92.28(18)$ \\
$\mathrm{N}(1)-\mathrm{W}-\mathrm{N}(4)$ & $80.85(15)$ \\
$\mathrm{W}-\mathrm{C}(1)-\mathrm{O}(1)$ & $173.9(5)$ \\
$\mathrm{W}-\mathrm{C}(2)-\mathrm{O}(2)$ & $177.1(5)$ \\
$\mathrm{W}-\mathrm{C}(3)-\mathrm{O}(3)$ & $175.7(5)$ \\
$\mathrm{W}-\mathrm{C}(4)-\mathrm{O}(4)$ & $173.9(5)$ \\
$\mathrm{W}(6)-\mathrm{C}(5)-\mathrm{C}(12)$ & $126.6(5)$ \\
$\mathrm{C}(6)-\mathrm{C}(5)-\mathrm{N}(1)$ & $111.6(5)$ \\
$\mathrm{C}(12)-\mathrm{C}(5)-\mathrm{N}(1)$ & $121.8(5)$ \\
$\mathrm{C}(5)-\mathrm{C}(6)-\mathrm{C}(7)$ & $106.1(5)$ \\
$\mathrm{C}(5)-\mathrm{C}(6)-\mathrm{C}(13)$ & $126.5(6)$ \\
$\mathrm{C}(7)-\mathrm{C}(6)-\mathrm{C}(13)$ & $127.4(6)$
\end{tabular}

(b) Compound 7

(i) Bond lengths

$\mathrm{W}-\mathrm{N}(1)$

$\mathrm{W}-\mathrm{N}(2)$

$\mathrm{W}-\mathrm{C}(1)$

$\mathrm{W}-\mathrm{C}(2)$

$\mathrm{W}-\mathrm{C}(3)$

W-C(4)

$\mathrm{N}(1)-\mathrm{N}(3)$

$\mathrm{N}(1)-\mathrm{C}(12)$

$\mathrm{N}(2)-\mathrm{N}(4)$

$\mathrm{N}(2)-\mathrm{C}(22)$

$\mathrm{N}(3)-\mathrm{C}$

$N(3)-C(14)$

$\mathrm{N}(4)-\mathrm{C}$

(ii) Bond angles

$\mathrm{N}(1)-\mathrm{W}-\mathrm{N}(2)$

$\mathrm{N}(1)-\mathrm{W}-\mathrm{C}(1)$

N(1)-W C(2)

$\mathrm{N}(1)-\mathrm{W}-\mathrm{C}(3)$

$\mathrm{C}(6)-\mathrm{C}(13)$
$\mathrm{C}(7)-\mathrm{C}(14)$
$\mathrm{C}(7)-\mathrm{N}(2)$
$\mathrm{C}(8)-\mathrm{N}(2)$
$\mathrm{C}(8)-\mathrm{N}(3)$
$\mathrm{C}(9)-\mathrm{C}(10)$
$\mathrm{C}(9)-\mathrm{C}(15)$
$\mathrm{C}(9)-\mathrm{N}(4)$
$\mathrm{C}(10)-\mathrm{C}(11)$
$\mathrm{C}(10)-\mathrm{C}(16)$
$\mathrm{C}(11)-\mathrm{C}(17)$
$\mathrm{C}(11)-\mathrm{N}(3)$
$\mathrm{N}(1)-\mathrm{N}(2)$
$\mathrm{N}(3)-\mathrm{N}(4)$

$1.491(8)$

$1.493(8)$

1.351(7)

$1.434(7)$

$1.441(6)$

$1.395(7)$

$1.492(8)$

$1.335(6)$

$1.356(8)$

1.493(7)

$1.479(8)$

$1.342(6)$

$1.390(6)$

$1.370(6)$

\section{C(6) $-\mathrm{C}(7)-\mathrm{C}(14)$}

$\mathrm{C}(6)-\mathrm{C}(7)-\mathrm{N}(2)$

C(14)-C(7)-N(2)

$\mathrm{N}(2)-\mathrm{C}(8)-\mathrm{N}(3)$

C(10)-C(9)-C(15)

C(10)-C(9)-N(4)

C(15)-C(9)-N(4)

$\mathrm{C}(9)-\mathrm{C}(10)-\mathrm{C}(11)$

$C(9)-C(10)-C(16)$

$C(11)-C(10)-C(16)$

$\mathrm{C}(10)-\mathrm{C}(11)-\mathrm{C}(17)$

( (10) $-C(11)-N(3)$

$C(17)-C(11)-N(3)$

$\mathrm{W}-\mathrm{N}(1)-\mathrm{C}(5)$

$\mathrm{W}-\mathrm{N}(1)-\mathrm{N}(2)$

C(5)-N(1)-N(2)

$\mathrm{C}(7)-\mathrm{N}(2)-\mathrm{C}(8)$

$\mathrm{C}(7)-\mathrm{N}(2)-\mathrm{N}(1)$

C(8)-N(2)-N(1)

$\mathrm{C}(8)-\mathrm{N}(3)-\mathrm{C}(11)$

$\mathrm{C}(8)-\mathrm{N}(3)-\mathrm{N}(4)$

C(11) $-\mathrm{N}(3)-\mathrm{N}(4)$

$\mathrm{W}-\mathrm{N}(4)-\mathrm{C}(9)$

$\mathrm{W}-\mathrm{N}(4)-\mathrm{N}(3)$

$\mathrm{C}(9)-\mathrm{N}(4)-\mathrm{N}(3)$

$131.0(5)$

$106.9(5)$

122.1(5)

112.4(4)

$127.9(5)$

$110.4(5)$

$121.7(5)$

106.1(4)

125.9(5)

$128.0(5)$

$129.7(5)$

$107.4(4)$

$122.9(5)$

$133.7(4)$

121.7(3)

104.3(4)

130.1(4)

111.2(4)

$118.7(4)$

130.2(4)

$118.5(4)$

111.2(4)

132.5(3)

122.2(3)

104.9(4)

$1.360(9)$
$1.178(10)$
$1.144(9)$
$1.131(9)$
$1.134(9)$
$1.442(9)$
$1.407(10)$
$1.375(10)$
$1.502(10)$
$1.486(11)$
$1.394(11)$
$1.359(11)$
$1.492(12)$

$\begin{array}{cl}78.35(19) & \mathrm{C}-\mathrm{N}(3)-\mathrm{C}(14) \\ 177.9(3) & \mathrm{N}(2)-\mathrm{N}(4)-\mathrm{C} \\ 97.91(24) & \mathrm{N}(2)-\mathrm{N}(4)-\mathrm{C}(24) \\ 93.7(3) & \mathrm{C}-\mathrm{N}(4)-\mathrm{C}(24)\end{array}$

$128.8(6)$

$119.6(5)$

$110.7(5)$

$129.7(6)$
TABLE 3 (continued)

\begin{tabular}{lrll}
\hline $\mathrm{N}(1)-\mathrm{W}-\mathrm{C}(4)$ & $93.8(3)$ & $\mathrm{N}(3)-\mathrm{C}-\mathrm{N}(4)$ & $110.7(5)$ \\
$\mathrm{N}(2)-\mathrm{W}-\mathrm{C}(1)$ & $101.1(3)$ & $\mathrm{W}-\mathrm{C}(1)-\mathrm{O}(1)$ & $173.7(6)$ \\
$\mathrm{N}(2)-\mathrm{W}-\mathrm{C}(2)$ & $91.75(24)$ & $\mathrm{W}-\mathrm{C}(2)-\mathrm{O}(2)$ & $171.7(6)$ \\
$\mathrm{N}(2)-\mathrm{W}-\mathrm{C}(3)$ & $94.6(3)$ & $\mathrm{W}-\mathrm{C}(3)-\mathrm{O}(3)$ & $170.4(7)$ \\
$\mathrm{N}(2)-\mathrm{W}-\mathrm{C}(4)$ & $171.7(3)$ & $\mathrm{W}-\mathrm{C}(4)-\mathrm{O}(4)$ & $178.7(7)$ \\
$\mathrm{C}(1)-\mathrm{W} \cdot \mathrm{C}(2)$ & $84.1(3)$ & $\mathrm{N}(1) \mathrm{C}(12) \mathrm{C}(11)$ & $123.5(6)$ \\
$\mathrm{C}(1)-\mathrm{W}-\mathrm{C}(3)$ & $84.3(3)$ & $\mathrm{N}(1)-\mathrm{C}(12)-\mathrm{C}(13)$ & $109.5(6)$ \\
$\mathrm{C}(1)-\mathrm{W}-\mathrm{C}(4)$ & $86.9(3)$ & $\mathrm{C}(11)-\mathrm{C}(12)-\mathrm{C}(13)$ & $127.0(7)$ \\
$\mathrm{C}(2)-\mathrm{W}-\mathrm{C}(3)$ & $167.7(3)$ & $\mathrm{C}(12)-\mathrm{C}(13)-\mathrm{C}(14)$ & $106.7(6)$ \\
$\mathrm{C}(2)-\mathrm{W}-\mathrm{C}(4)$ & $86.7(3)$ & $\mathrm{N}(3)-\mathrm{C}(14)-\mathrm{C}(13)$ & $106.5(6)$ \\
$\mathrm{C}(3)-\mathrm{W}-\mathrm{C}(4)$ & $88.5(3)$ & $\mathrm{N}(3)-\mathrm{C}(14)-\mathrm{C}(15)$ & $122.7(6)$ \\
$\mathrm{W}-\mathrm{N}(1)-\mathrm{N}(3)$ & $121.6(4)$ & $\mathrm{C}(13)-\mathrm{C}(14)-\mathrm{C}(15)$ & $130.8(7)$ \\
$\mathrm{W}-\mathrm{N}(1)-\mathrm{C}(12)$ & $132.7(4)$ & $\mathrm{N}(2)-\mathrm{C}(22)-\mathrm{C}(21)$ & $122.2(7)$ \\
$\mathrm{N}(3)-\mathrm{N}(1)-\mathrm{C}(12)$ & $105.7(5)$ & $\mathrm{N}(2)-\mathrm{C}(22)-\mathrm{C}(23)$ & $110.2(6)$ \\
$\mathrm{W}-\mathrm{N}(2)-\mathrm{N}(4)$ & $121.1(4)$ & $\mathrm{C}(21)-\mathrm{C}(22)-\mathrm{C}(23)$ & $127.6(7)$ \\
$\mathrm{W}-\mathrm{N}(2)-\mathrm{C}(22)$ & $132.5(5)$ & $\mathrm{C}(22)-\mathrm{C}(23)-\mathrm{C}(24)$ & $106.4(6)$ \\
$\mathrm{N}(4)-\mathrm{N}(2)-\mathrm{C}(22)$ & $105.4(5)$ & $\mathrm{N}(4)-\mathrm{C}(24)-\mathrm{C}(23)$ & $107.3(7)$ \\
$\mathrm{N}(1)-\mathrm{N}(3)-\mathrm{C}$ & $119.3(5)$ & $\mathrm{N}(4)-\mathrm{C}(24)-\mathrm{C}(25)$ & $123.3(7)$ \\
$\mathrm{N}(1)-\mathrm{N}(3)-\mathrm{C}(14)$ & $111.5(5)$ & $\mathrm{C}(23)-\mathrm{C}(24)-\mathrm{C}(25)$ & $129.4(7)$ \\
\hline
\end{tabular}

$120.8^{\circ}$ in $6,68.2^{\circ}$ in 7 and $68.1^{\circ}$ in 8. Apparently, the boat conformation does not change much whether the central metal atom is tungsten or molybdenum. This feature is to be expected from the similarity in the radii of the two atoms because of the lanthanide contraction [15]. However, when the hydrogen atom on the ring-4 position of $\mathrm{Pz}^{\prime}$ is replaced by a methyl group, the conformation changed greatly as observed in various angles for 6 . We found that the net result from this conformational modification is best exemplified by one angle, formed by two cis-carbonyl groups and the central metal atom, which is $170.80(21)^{\circ}$ in $6,167.7(3)^{\circ}$ in 7 , or $167.3(1)^{\circ}$ in 8 . As observed in Table 3, either boat conformation resulting from different substituents has

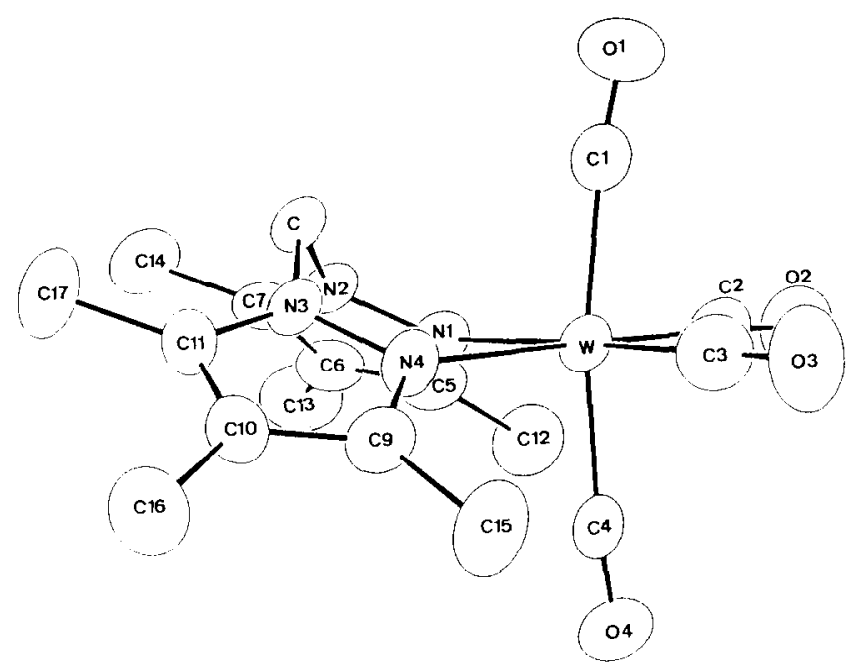

Fig. 2. ORTEP drawing of $\left[\mathrm{W}\left\{\eta^{2}-\left(\mathrm{H}_{2} \mathrm{CPz}_{2}^{\prime \prime}\right)\right\}(\mathrm{CO})_{4}\right](6)$ with the numbering scheme. Thermal ellipsoids are drawn at the $50 \%$ probability level. 


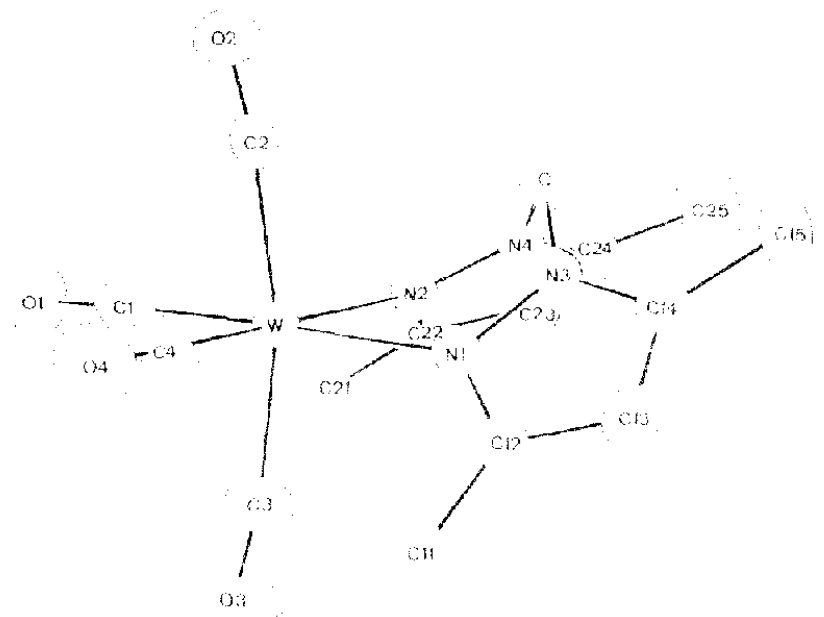

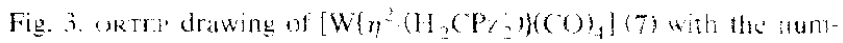

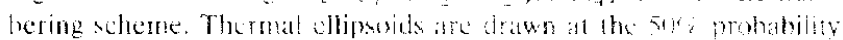
level.

caused some bond angles. especially those formed by carbonyls in the molecule, at the corresponding locations to differ significantly from each other: for example. $C(3)-W-C(4) 89.71(25)^{\circ}$ in 6 and $(13)-W-(1)$ $84.3(3)^{\circ}$ in 7 (c). Figs. 2 and 3 ), and influences (probably in a complicated way) the celevant artwonyl stretching frequencies so that no simple trend in the w(CO) values can he found. How can we explatin the structural change observed as more substituents are added? Constructing a model shows clearly that nombonded repulsive interactions exist between the suth situents especially at the ring-3 and ring 5 ) pustions of two pyrazolyl rings as well an interachoms hetween the substituents at the ring-3 (or ring 5 ) and at the ring-4 positions of the same pyrazolyl ring. Hence. when the replacement by nethyl groups af the bydrogen atoms at the ring-4 positions of $11,6 \mathrm{P} L$ aceurs, repulsive interactions increase. creating a poorer environment for the nonbonded interations beween lwo

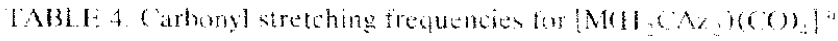

\begin{tabular}{|c|c|c|c|c|c|}
\hline$M$ & $\mathrm{Nz}$ & A: & $B_{1}$ & $A_{i}$ & $\mathrm{~B}_{2}$ \\
\hline $\mathrm{Cr}$ & $P \%$ & 31616 & 1890 & 1972 & 8833 \\
\hline $\mathrm{Cr}$ & $\mathrm{P} \%$ & $21 / 10$ & $18 \times 7$ & 1867 & $\mid 630$ \\
\hline $\mathrm{Cr}$ & $P$, & 2014 & 1884 & ixhy & 18.77 \\
\hline Mo & $P_{\%}$ & 20111 & 1 sok & 4875 & $1 \times 37$ \\
\hline Mo & $1 \%$ & 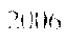 & 1503 & $1 \times 2$ & $\mid \times 3+4$ \\
\hline Mo & $\mathrm{PH}^{\prime \prime}$ & 2914 & 1805 & $1 \times 74$ & 1832 \\
\hline$w$ & $P_{Z}$ & 20104 & 1874 & $\ldots !$ & M? \\
\hline W & $P t$ & 20130 & $1,7 ?$ & 1362 & 1528 \\
\hline$W$ & {$[\% ;$} & 20103 & 1077 & 1867 & 1922 \\
\hline
\end{tabular}

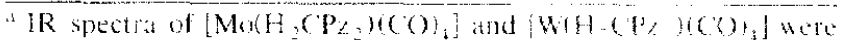
me:asured in Mers. Thowe of all onthe compleses bere meanded in

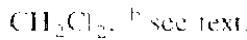

methyl groups at the ring-3 for ring-5) positions of two different pyrazolyl rings in $\mathrm{H}_{2} \mathrm{CP}_{2}$ ", as indicited by the large angle $(120,8)$ in 6 . bomed by two pyratyl-ring

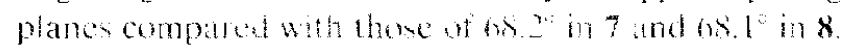

In conctusion. the flextility of the had six-membered metallade allow adjusment of the chelate structure to minmize the nombuted epulsive interatoms. The streturat modification then atrers the clec-

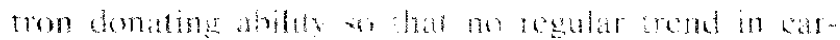

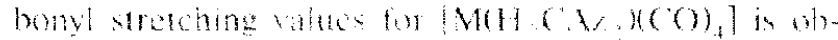
served as more rophatment of hadroen atoms by methytgromp stortineme take place on the pyrably ring, the clectom domating athility of the resulting lig-

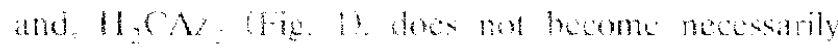

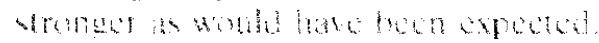

\section{Acknowledgment}

Wo wish to thank the Vutional Science Council of the Republic of China for financial support of this

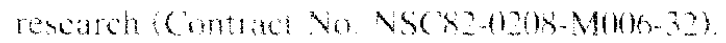

\section{References}

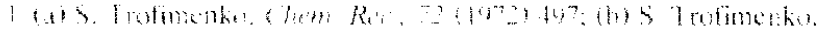

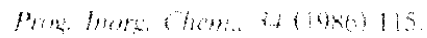

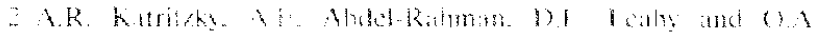

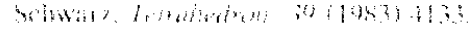

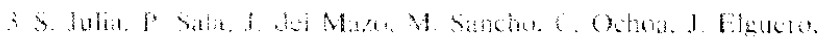

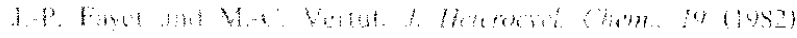
$1,1.1$

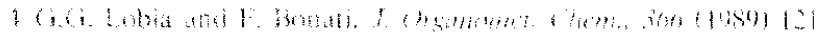

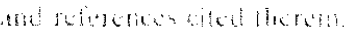

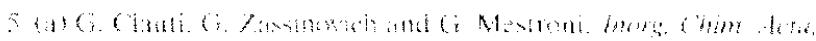
If a (bent

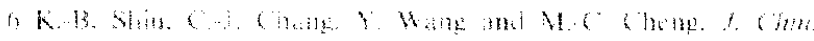

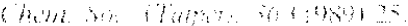

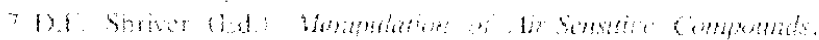

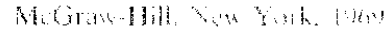

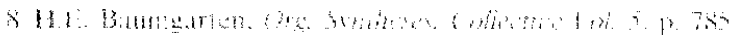

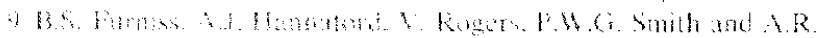

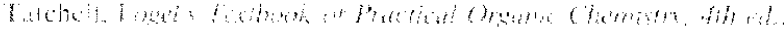

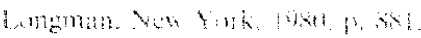

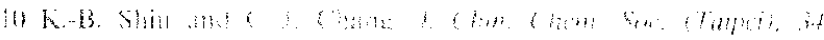
(1)

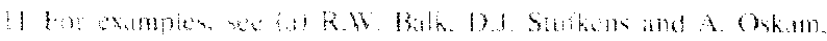

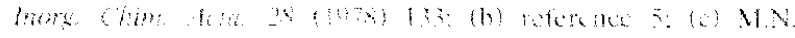

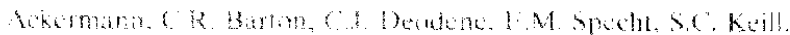

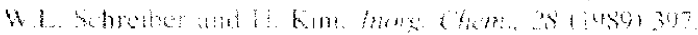

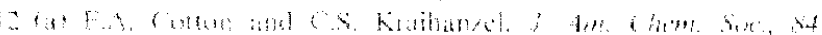

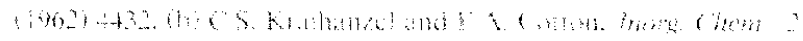
490,33

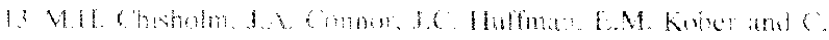

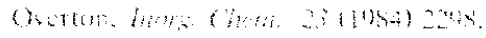

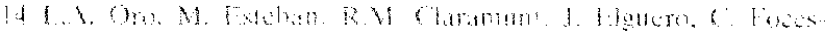

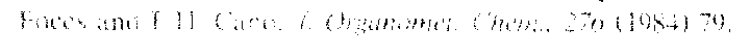

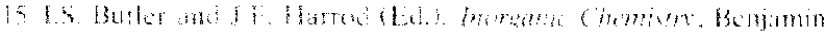

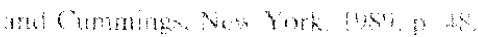

